\title{
Dynamics of Changes in Selected Soil Traits in the Profiles of Arable Soils Anthropogenically Alkalised by the Cement and Lime Industry within the Kielecko-Łagowski Vale (Poland)
}

\author{
Anna Świercz ${ }^{1, *(\mathbb{D}}$, Agnieszka Gandzel $^{2}$ and Ilona Tomczyk-Wydrych ${ }^{3}[$ \\ 1 Institute of Geography and Environmental Sciences, Jan Kochanowski University, 7 Uniwersytecka Street, \\ 25-406 Kielce, Poland \\ 2 Technical and Industry Training Center, S. Leszczyńskiej Street 8, 32-600 Oświęcim, Poland; a.gandzel@op.pl \\ 3 Institute of Geography and Environmental Sciences, Doctoral School, Jan Kochanowski University, \\ 7 Uniwersytecka Street, 25-406 Kielce, Poland; ilonatomczyk@interia.eu \\ * Correspondence: swierczag@poczta.onet.pl
}

Citation: Świercz, A.; Gandzel, A.; Tomczyk-Wydrych, I. Dynamics of Changes in Selected Soil Traits in the Profiles of Arable Soils

Anthropogenically Alkalised by the Cement and Lime Industry within the Kielecko-Łagowski Vale (Poland). Land 2021, 10, 84. https://doi.org/ 10.3390/land10010084

Received: 9 December 2020

Accepted: 14 January 2021

Published: 18 January 2021

Publisher's Note: MDPI stays neutral with regard to jurisdictional claims in published maps and institutional affiliations.

Copyright: (c) 2021 by the authors. Licensee MDPI, Basel, Switzerland. This article is an open access article distributed under the terms and conditions of the Creative Commons Attribution (CC BY) license (https:// creativecommons.org/licenses/by/ $4.0 /)$.

\begin{abstract}
This study presents the influence of the cement and lime industry on the physical and chemical properties of arable soils. In spite of using modern forms of environmental protection against dust emissions, this type of industry causes unfavourable phenomenon of excessive alkalisation of soil. This process is relatively rare in Poland. However, in the Świętokrzyskie Province, it has been responsible for the largest transformation of soils in recent years. The analysis included soil samples taken from five profiles located in the vicinity of Dyckerhoff Polska Sp. z o.o. Nowiny Cement Plant. The study results obtained in 2019 were compared with those obtained in 1978 and 2005. The most attention was paid to soil $\mathrm{pH} ; \mathrm{CaCO}_{3}$ content; organic carbon and nitrogen content; concentrations of available components such as $\mathrm{P}_{2} \mathrm{O}_{5}, \mathrm{~K}_{2} \mathrm{O}$ and $\mathrm{Mg}$; and the saturation level of sorption complex with alkaline cations. It was found that long-term imission of pollutants caused significant changes in the basic soil properties, which remain in soils despite the evident decrease in the cement-lime dust emission. These include high $\mathrm{pH}$ values, excessive $\mathrm{CaCO}_{3}$ content, high soil saturation with alkaline cations and decreases in total carbon content, which were especially visible in soil humus horizons.
\end{abstract}

Keywords: alkalisation; cement plant; arable soils; physicochemical properties

\section{Introduction}

Soils fulfil a number of natural and economic functions [1-3]. The production function is generally mentioned first, where soils are a substrate for the cultivation of food, forage and industrial crops or for forest plantations. In this context, the long-term monitoring of the basic properties of soils plays an important role in tracking the dynamics of changes under the influence of anthropogenic pressures changing over time. In addition, the condition of soils may directly and indirectly affect the responses of plant and animal organisms to the improvement of soil quality or its degradation $[4,5]$.

The well-developed cement and lime industry causes far-reaching transformations of soil [6-15]. Pollutants are emitted in the form of dusts and gases, which in turn causes their release to all components of the environment, including soils [7,8,10,12].

The chemical composition of cement and lime dust is relatively constant and depends on the composition of raw materials used in technological processes. The emitted dust contains mainly $\mathrm{CaO}(<43 \%)$ and is characterised by high $\mathrm{pH}_{\mathrm{KCl}}$ values $(<12.5)$ in terms of alkaline $\mathrm{pH}$, which, due to many years of emission, significantly affects the properties of soil [12,15-18]. Moreover, it is more often indicated that the excessive deposition of cement and lime dust into soils is a highly stressful factor causing acute physiological reactions in plants, including the reduction of photosynthesis and gas exchange [7,19].

In the Świętokrzyskie Province, the cement and lime industry is characterised by dynamic development, and its level of technological achievement, production volume and 
raw material extraction scale is among the leading ones in Europe. Long-term alkaline pressure on the soils located in the area of $500 \mathrm{~km}^{2}$ nearby the Dyckerhoff Polska Sp. z o.o. Nowiny Cement Plant, "Trzuskawica" S.A. Cement Roadstone Holding, "Jaźwica" Mineral Resource Mine, "Lafarge" Dolomite Mine in Radkowice, Lafarge Cement Plant in Małogoszcz and Lhoist S.A. Lime Production Plant in Bukowa has been lasting and thoroughly influencing the basic physicochemical properties of the soils, especially the Haplic Podzol soils formed on sands, since the 1960s. Due to modernisation, up-to-date methods of management and control of a technological process have been introduced, considerably reducing the negative impact on the environment. However, in spite of restricted emission, lime plants are the main source of cement and lime dust emissions that contain a compound of silicate, lime and aluminium minerals. Moreover, during thermal processes occurring in a rotary kiln while producing cement, $\mathrm{CO}_{2}$ and $\mathrm{SO}_{2}$ are emitted into the atmosphere. In the case of lime production, a large amount of $\mathrm{CaO}$ and $\mathrm{CO}_{2}$ is released. Significant amounts of calcium ions entering the soil cause the effect of "over-fertilization"-alkalisation which can chemically degrade soils [15,17,19-22]. Although soil alkalisation is relatively rare in Poland, it is this process which has been responsible for the largest transformation of soils in the Świętokrzyskie Province in recent years.

The aim of the study was to reveal changes of particular physicochemical properties of the arable soils located in the monitored areas of the Kielecko-Łagowski Vale between 1978 and 2019 as a zone of long-term and declining alkaline emission. The following research hypotheses were verified: Whether the reduced alkaline emission has an effect on the soil properties in terms of its regeneration (e.g., decrease in $\mathrm{pH}$, hydrolytic acidity, carbonate content), which soil type or genetic level will react most strongly to the reduced pressure and whether the observed trends can be considered as permanent.

\section{Materials and Methods}

\subsection{Study Area}

The research site covered an area of the Sitkówka Nowiny Commune, which lies within the Świettokrzyskie Mountains mesoregion belonging to the Kielce Upland macroregion. The geological structure is diversified. The subsoil is dominated by Cambrian and Devonian quartzites, Cretaceous and Jurassic limestones and Triassic sandstones, as well as slates and clay. The highest hills do not exceed $388 \mathrm{~m}$ above sea level. Sitkówka-Nowiny is an industrial and agricultural commune with an average population of 170 people per $\mathrm{km}^{2}$. The agricultural land occupies $42 \%$ of the commune and $37 \%$ of the forests. Due to the dustiness of land, especially the aboveground part of plants and disruption of water relations, the agricultural land cannot be used intensively. This problem also applies to arable soils, which are not used according to their agricultural suitability, because excessive dustiness of calcium and sulphur compounds interfere with the vegetation of open-pollinated plants and cause degradation of aboveground parts of most crops. The alkaline reaction of soils formed as a result of long-term precipitation of calcium compounds creates limitations in the cultivation of weak soils, which dominate in the Sitkówka-Nowiny Commune, especially rye, potatoes, lupine, serradella and oats. Wheat, barley and legume plants, which yield better in an alkaline environment, are necessarily dominant in the crop structure [23].

The climate is moderate. Taking into account the thermal classification of the years, since 1971, we have observed a constant increase in the average annual temperature. The year 1978, according to $H$. Lorenc classification (2000), could be considered as cold $\left(t_{s r}-1.0 \sigma \leq t_{z}<t_{s i r}-0.5 \sigma\right)$, the year 2005 as slightly warm $\left(t_{s i r}+0.5 \sigma<t_{z} \leq t_{s r}+1.0 \sigma\right)$, the year 2019 as extremely warm $\left(t_{z}<t_{s i r}-2.5 \sigma\right)$ (where $t_{s ́ r}=$ temperature from the years 1971-2000, $t_{z}=$ temperature in a given year, $\sigma=$ standard deviation). Therefore, over the past 40 years, an increase in the average annual temperature of almost $2{ }^{\circ} \mathrm{C}$ was observed (the average annual temperature in 1978 was $7.10^{\circ} \mathrm{C}$; in $2005,7.8{ }^{\circ} \mathrm{C}$; in 2019 , $9.4^{\circ} \mathrm{C}$ ). It is worth noting that, since 2004 , extreme temperatures exceeding $30^{\circ} \mathrm{C}$ have been observed in June and August, while humidity conditions, despite significant fluctuations, 
are considered comparable. The winds have been characterized by an average velocity of $3 \mathrm{~m} / \mathrm{s}$, predominantly from the western and north-western directions $(70 \%)[24,25]$.

In June 2019, five soil profiles were collected in order to identify the types of soils and their physicochemical properties (the same areas were taken into account in 1978 and then in 2005). The profiles were located at a distance from $400 \mathrm{~m}$ to $3 \mathrm{~km}$ away from the Dyckerhoff Polska Sp. z o.o. Nowiny Cement Plant (Figure 1), in the area of excessive cement and lime dust emission into soils (Profile no. 1-3 km; Profile no. 2-2.2 km; Profile no. 3-1.7 km; Profile no. 4-2.5 km; Profile no. 5-400 m).

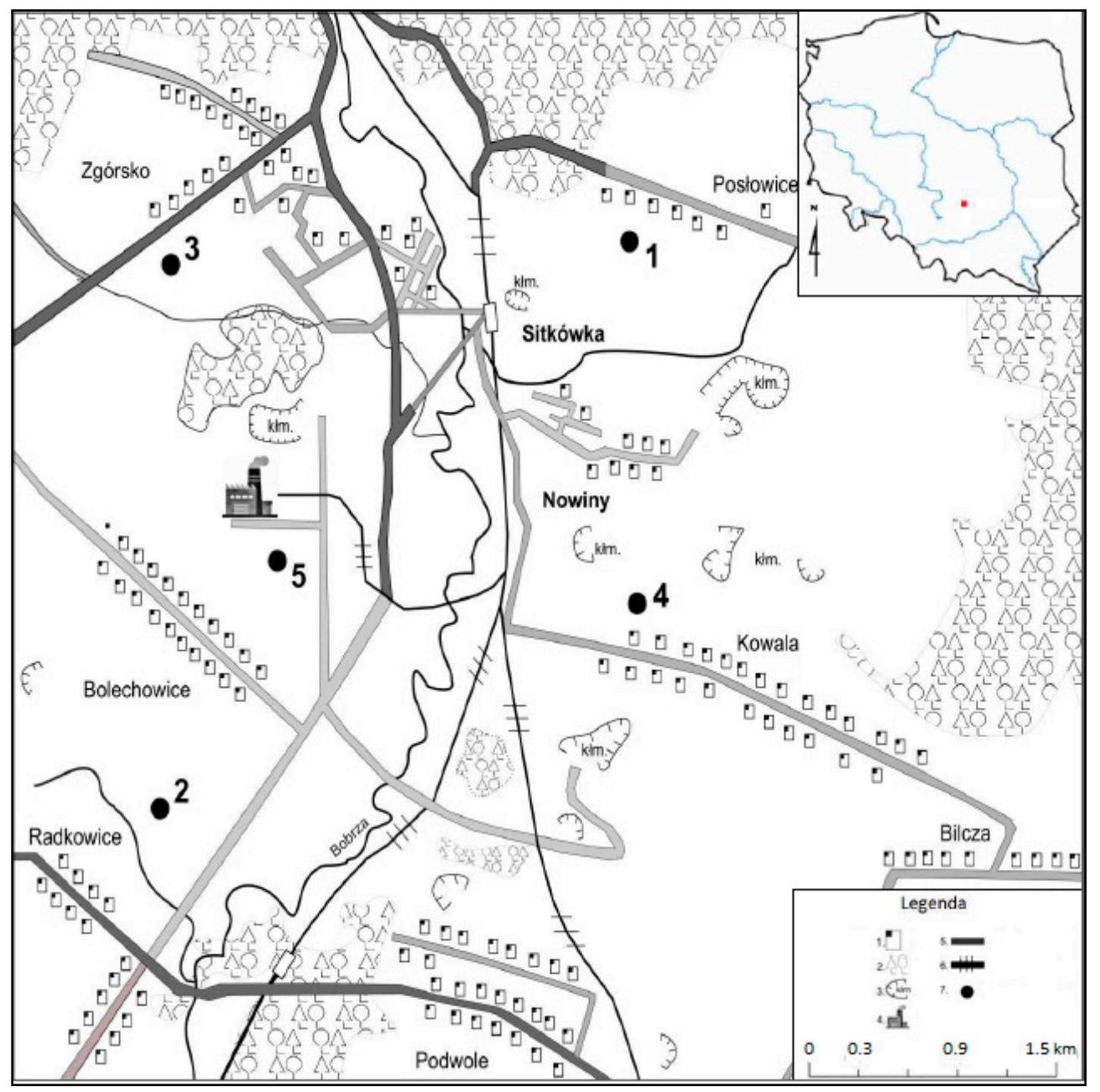

Figure 1. Location of soil pits depending on the distance from the dust emitter (Nowiny Cement Plant Sp. z o. o.). Key: (1) Buildings; (2) forests; (3) quarries; (4) cement plants; (5) roads; (6) railways; (7) soil pits.

Soil samples were taken each time in June. Soil pits up to the depth of 40-120 cm (depending on the soil type) were made onsite. Soil samples of damaged structures (about $1 \mathrm{~kg}$ ) were taken from each level according to the methods used in soil science.

\subsection{Data Collection and Analysis}

Soil profiles were described in detail according to the methodology as it has been done in the past years [26]. During fieldwork, the thickness of individual subhorizons, grain size composition, colour based on the Standard Soil Colour Charts according to Munsell and the type of soil humus were determined. Samples for research were collected from each genetic subhorizon. In each profile, soil material with disturbed structure was collected from the distinguished genetic subhorizon, which was used for analytical laboratory work. The soil samples were dried at room temperature to an air-dry state. After drying, 
the mineral samples were ground in a porcelain mortar using a pestle and then sieved through a soil sieve with a mesh diameter of $1 \mathrm{~mm}$. Soil samples were crushed with a Fitch laboratory grind (A-11 model). The samples prepared in this way were then put in cardboard boxes with laboratory metrics. Each soil sample taken from selected genetic horizons, and subhorizons was examined on the basis of the methodology proposed by Kowalkowski and Swałdek (1991) [27] and that by Karczewska and Kabała (2008) [28]:

- A grain size composition of earthy parts by sieve analysis and Casagrande's aerometric method modified by Prószyński [27,28];

- $\quad$ Soil reaction by potentiometric titration $\left(\mathrm{pH} \mathrm{H}_{2} \mathrm{O}, \mathrm{pH}_{\mathrm{KCl}}\right)[27,28]$;

- Organic carbon $\left(\mathrm{C}_{\text {org }}\right.$ ) by Tiurin's methods (for mineral levels of soils with $\mathrm{C}_{\text {org }}<15 \%$ method consisted in oxidation of carbon with $0.4 \mathrm{~N}$ solution $\mathrm{K}_{2} \mathrm{Cr}_{2} \mathrm{O}_{7}$ (in relation to $\mathrm{Ag}_{2} \mathrm{SO}_{4}$ catalyst) in acidic medium, the residue of which was titrated with Mohr's salt $\left(0.1 \mathrm{~N} \mathrm{Fe}\left(\mathrm{NH}_{2}\right) \mathrm{SO}_{4} \cdot 6 \mathrm{H}_{2} \mathrm{O}\right)$ in relation to orthophenanthroline. For humus levels with $\mathrm{C}_{\text {org }}>15 \%$, the Alten method was used [27,28];

- Nitrogen in general $\left(\mathrm{N}_{\text {org }}\right)$ by modified Kjeldahl's method in Kjeltec Auto Analyzer [27,28];

- Hydrolytic acidity $\mathrm{Hh}$ by Kappen's method in $0.5 \mathrm{M} \mathrm{Ca}\left(\mathrm{CH}_{3} \mathrm{COO}\right)_{2}$ solution $[27,28]$;

- Sum of exchangeable basic cations (S) by Kappen's method in $0.5 \mathrm{M} \mathrm{NH}_{4} \mathrm{Cl}$ solution at $\mathrm{pH} 8.2$ [27,28];

- Acidity $\mathrm{Hw}$ and exchangeable aluminium $\mathrm{Al}^{3+}$ by Sokołow's method [27,28];

- Content of ingestible ingredients $\mathrm{P}_{2} \mathrm{O}_{5}, \mathrm{~K}_{2} \mathrm{O}, \mathrm{Mg}$ by Enger-Rhiem's method PN-R$04023\left(\mathrm{P}_{2} \mathrm{O}_{5}\right), \mathrm{PN}-\mathrm{R} 04022\left(\mathrm{~K}_{2} \mathrm{O}\right), \mathrm{PN}-\mathrm{R}-04024(\mathrm{Mg})[27,28]$.

Dust fall of particle matter was studied using a trapping measure (Bergerhoff type). Glass funnels (input diameter: $7 \mathrm{~cm}$, outlet diameter: $2 \mathrm{~cm}$ ), connected to the bottles and exposed at 5 research positions, were used in February, May, August and November. Afterward, aggregated rainwater was evaporated, and funnels and jars were rinsed thoroughly with hexane. Ashless cellulose drains were used to extract dust from the mixture. After the exposure, the drains were being dried for $2 \mathrm{~h}$ at 45 degrees Celsius and then weighed. Amount of dust fall was calculated by mass difference, time of exposure and funnel area [29] (Table 1, Figure 2).

All analytical procedures were identical to those applied in 1978 and in 2005 in order to make the study results compliant and comparable [30].

Table 1. Amount of dust fall at selected research positions per $\mathrm{g} / \mathrm{m}^{2}$.

\begin{tabular}{|c|c|c|c|c|c|c|c|c|}
\hline \multirow[b]{2}{*}{$\begin{array}{c}\text { Area } \\
\text { No. }\end{array}$} & \multirow[b]{2}{*}{ Longitude } & \multirow[b]{2}{*}{ Latitude } & \multicolumn{2}{|c|}{1978} & \multicolumn{2}{|c|}{2005} & \multicolumn{2}{|c|}{2019} \\
\hline & & & $\begin{array}{c}\text { Mean } \\
\text { Value } \\
\text { (Min-Max) }\end{array}$ & $\begin{array}{l}\text { Annual } \\
\text { Value }\end{array}$ & $\begin{array}{c}\text { Mean } \\
\text { Value } \\
\text { (Min-Max) }\end{array}$ & $\begin{array}{l}\text { Annual } \\
\text { Value }\end{array}$ & $\begin{array}{c}\text { Mean } \\
\text { Value } \\
\text { (Min-Max) }\end{array}$ & $\begin{array}{c}\text { Annual } \\
\text { Value }\end{array}$ \\
\hline $\begin{array}{c}\text { Profile } 1 \\
\text { Posłowice }\end{array}$ & 20.574921 & 50.822278 & $\begin{array}{c}18.6 \\
(15.7-55.1)\end{array}$ & 245.6 & $\begin{array}{c}3.01 \\
(2.0-11.7)\end{array}$ & 47.3 & $\begin{array}{c}2.9 \\
(2.1-11.7)\end{array}$ & 45.2 \\
\hline $\begin{array}{l}\text { Profile } 2 \\
\text { Jesionki }\end{array}$ & 20.525097 & 50.780045 & $\begin{array}{c}28.3 \\
(11.1-67.8) \\
\end{array}$ & 340.5 & $\begin{array}{c}6.3 \\
(1.5-17.3) \\
\end{array}$ & 62.0 & $\begin{array}{c}6.1 \\
(2.1-14.7) \\
\end{array}$ & 60.1 \\
\hline $\begin{array}{l}\text { Profile } 3 \\
\text { Zgórsko }\end{array}$ & 20.517237 & 50.827315 & $\begin{array}{c}12.9 \\
(6.6-20.8) \\
\end{array}$ & 190.7 & $\begin{array}{c}2.9 \\
(1.5-9.5) \\
\end{array}$ & 35.2 & $\begin{array}{c}2.7 \\
(1.7-10.1) \\
\end{array}$ & 33.1 \\
\hline $\begin{array}{l}\text { Profile } 4 \\
\text { Kowala }\end{array}$ & 20.568548 & 50.801873 & $\begin{array}{c}53.1 \\
(14.6-91.9) \\
\end{array}$ & 497.5 & $\begin{array}{c}7.9 \\
(1.6-23.6) \\
\end{array}$ & 87.4 & $\begin{array}{c}7.8 \\
(1.5-19.3) \\
\end{array}$ & 89.2 \\
\hline $\begin{array}{l}\text { Profile } 5 \\
\text { Sitkówka }\end{array}$ & 20.515501 & 50.812118 & $\begin{array}{c}65.0 \\
(20.2-85.1)\end{array}$ & 651.4 & $\begin{array}{c}10.9 \\
(1.9-22.2)\end{array}$ & 111.5 & $\begin{array}{c}9.2 \\
(1.7-20.1)\end{array}$ & 100.4 \\
\hline
\end{tabular}




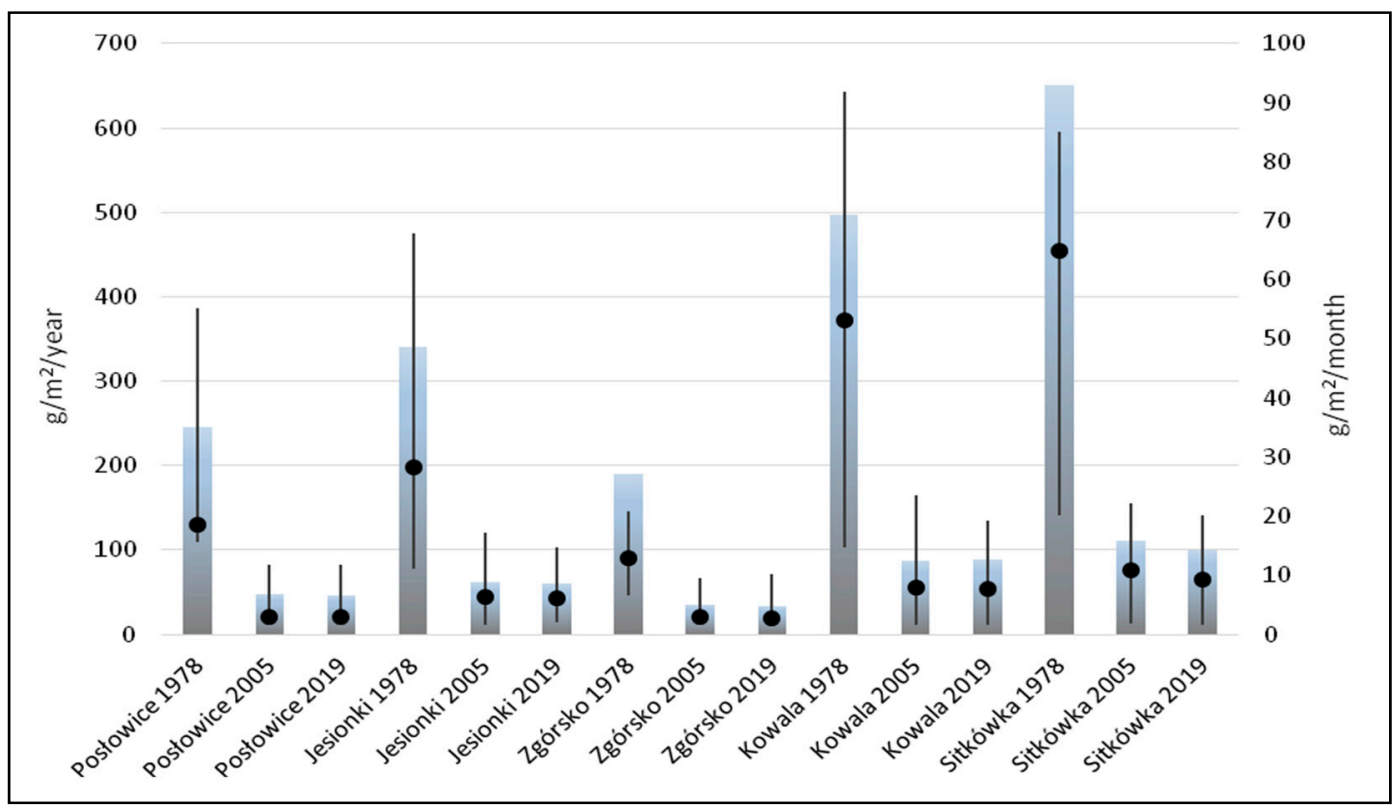

Figure 2. Dynamics of annual and monthly dust fall per $\mathrm{g} / \mathrm{m}^{2}$ in soil profiles at selected research positions in (1) Posłowice, (2) Jesionki, (3) Zgórsko, (4) Kowala, (5) Sitkówka.

\section{Results}

The main source of soil pollution in the abovementioned area is the Dyckerhoff Polska Sp. z o.o. Nowiny Cement Plant and a few other cement plants or quarries, such as the "Trzuskawica" S.A. Cement Roadstone Holding, "Jaźwica" Mineral Resource Mine, Morawica S.A. Limestone Mine, Kowala Quarry, "Lafarge" Dolomite Mine in Radkowice and Nordkalk Sp. z o.o. Miedzianka Production Plant.

In 1978, the annual lime-cement dust emission was systematically decreasing (from $190 \mathrm{~g} / \mathrm{m}^{2}$ to $33 \mathrm{~g} / \mathrm{m}^{2}$ in 2017). Average month-long dust fall tended to decrease as well (from $65 \mathrm{~g} / \mathrm{m}^{2}$ in 1978 to $9.2 \mathrm{~g} / \mathrm{m}^{2}$ in 2019). The biggest reduction (by around $85 \%$ ) of dust emission was found at research position no. 5 located in Sitkówka. At other positions, the decline in dust fall was equally high (around $82 \%$ ). The analysed soils were classified as different types [30] (Table 2).

Table 2. Profile structure and general characteristics of soils ( ${ }^{*}$ names of soils and sequences of horizons are given according to the Systematics of Polish Soils from 2011 and (World Reference Base for Soil Resources) WRB 2006, ** Grain size groups, according to the United States Department of Agriculture (USDA) and (Soil Science Society of Poland) PTG 2008).

\begin{tabular}{|c|c|c|c|c|c|}
\hline $\begin{array}{l}\text { Area } \\
\text { No. }\end{array}$ & $\begin{array}{l}\text { Horizontal } \\
\text { Composition }\end{array}$ & $\begin{array}{c}\text { Type } \\
\text { and Sub-Type * }\end{array}$ & Type & $\begin{array}{l}\text { Grain-Size } \\
\text { Groups ** }\end{array}$ & $\begin{array}{l}\text { Type of } \\
\text { Land Use }\end{array}$ \\
\hline $\begin{array}{l}\text { Profile } 1 \\
\text { Posłowice }\end{array}$ & Ap-Et-Bt-BC & Haplic Luvisol & loess & $\begin{array}{l}\text { silt } \\
\text { silt }\end{array}$ & arable land \\
\hline $\begin{array}{l}\text { Profile } 2 \\
\text { Jesionki }\end{array}$ & Ap-AC-2Cg-2G & Mollic Fluvisol & alluvial deposit & $\begin{array}{l}\text { silt covering loamy } \\
\text { sand }\end{array}$ & fields \\
\hline $\begin{array}{l}\text { Profile } 3 \\
\text { Zgórsko }\end{array}$ & Ap-Ees-Bhs-C & Haplic Podzol & fluvioglacial sands & $\begin{array}{l}\text { sand covering } \\
\text { medium and fine } \\
\text { sand }\end{array}$ & arable land \\
\hline $\begin{array}{l}\text { Profile } 4 \\
\text { Kowala }\end{array}$ & $\mathrm{Au}-\mathrm{AC}-\mathrm{Cg}$ & Mollic Gleysol & fluvial sands & loamy sand & $\begin{array}{l}\text { fields } \\
\text { the green use }\end{array}$ \\
\hline $\begin{array}{l}\text { Profile } 5 \\
\text { Sitkówka }\end{array}$ & Ap-ACca-Rca & Rendzic Leptosol & $\begin{array}{l}\text { Devonian } \\
\text { limestones }\end{array}$ & $\begin{array}{c}\text { clay loam covering } \\
\text { loam }\end{array}$ & arable land \\
\hline
\end{tabular}


There were no systematic differences between nowadays and the archival results. The names of soil horizons used in 1978 were merely adjusted to the contemporary standards [31]. Divergences in results of analysis of grain size composition were lower than $3-5 \%$ and stayed in the range of analytical error (Table 3).

Table 3. Grain size composition of analysed soils (wg PTG 2008 and USDA).

\begin{tabular}{|c|c|c|c|c|c|}
\hline \multirow[b]{2}{*}{ Profile No. } & & \multirow[b]{2}{*}{ Depth cm } & \multicolumn{3}{|c|}{ Diameter Limits $(\mathrm{mm})$ in \% } \\
\hline & & & 2-0.05 & 0.05-0.002 Silt & $\begin{array}{c}<0.002 \\
\text { Clay }\end{array}$ \\
\hline \multirow{4}{*}{1} & Ap & $0-15$ & 37 & 54 & 9 \\
\hline & Et & $15-45$ & 48 & 46 & 6 \\
\hline & $\mathrm{Bt}$ & $45-60$ & 50 & 42 & 8 \\
\hline & $\mathrm{BC}$ & $60-120$ & 23 & 67 & 10 \\
\hline \multirow{4}{*}{2} & Ap & $0-17$ & 50 & 45 & 5 \\
\hline & $\mathrm{AC}$ & $17-30$ & 73 & 21 & 6 \\
\hline & $2 \mathrm{Cg}$ & $30-50$ & 87 & 10 & 3 \\
\hline & $2 \mathrm{G}$ & $50-70$ & 70 & 15 & 15 \\
\hline \multirow{4}{*}{3} & Ap & $0-12$ & 94 & 5 & 1 \\
\hline & Ees & $12-28$ & 94 & 4 & 2 \\
\hline & Bhs & $28-48$ & 93 & 5 & 2 \\
\hline & $\mathrm{C}$ & $48-110$ & 95 & 4 & 1 \\
\hline \multirow{3}{*}{4} & $\mathrm{Au}$ & $0-16$ & 79 & 17 & 4 \\
\hline & $\mathrm{AC}$ & $16-55$ & 81 & 11 & 8 \\
\hline & $\mathrm{Cg}$ & 55-110 & 93 & 5 & 2 \\
\hline \multirow{3}{*}{5} & Ap & $0-10$ & 57 & 30 & 13 \\
\hline & ACca & $10-21$ & 60 & 15 & 25 \\
\hline & Rca & $21-35$ & - & - & - \\
\hline
\end{tabular}

The grain size composition of the analysed soils was diversified. These were soils composed of sand formations (profiles 3, 4), and others from dust formations (profiles 1,2) and clay ones (profile 5). Long-term emission of lime-cement dusts with high specific density $\left(>2.9 \mathrm{~g} \mathrm{~cm}^{-3}\right)$ [22] had no influence on changes in the grain size composition of the soils (even though their increase specific density on surface levels were featured in the literature, suggesting the phenomenon of aggregation of soil particle growth under the influence of intensive forest soils liming caused by strong "slurrying") [32].

The reaction of analysed soils was strictly connected to the variable deposition of alkaline dust. Changes in humus horizons appeared as follows: In 1978, $\mathrm{pH}_{\mathrm{KCl}}$ ranged from 6.11 to 7.30 ; then in 2005, it was 6.89 to 8.01 ; and in 2017, a growth of $\mathrm{pH}$ up to 8.21 was noticed, despite a decreasing trend of deposited alkaline dust (Table 4).

Table 4. Selected chemical characteristics of analysed soils.

\begin{tabular}{|c|c|c|c|c|c|c|c|c|c|c|c|c|c|c|c|}
\hline \multirow{2}{*}{ 㝵 } & \multirow{2}{*}{ 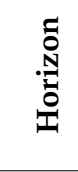 } & ص̃ & \multirow{2}{*}{ 올 } & \multirow{2}{*}{$\frac{\bar{v}}{2}$} & $\bigcup_{0}^{\infty}$ & ن் & 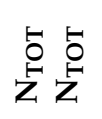 & \multirow[t]{2}{*}{ ت } & 寽 & $\infty$ & $H \cup$ & \multirow{2}{*}{$\frac{>\infty}{[\%]}$} & $\begin{array}{l}10 \\
0 \\
1\end{array}$ & $\begin{array}{l}\text { O } \\
\underline{v}\end{array}$ & \multirow[t]{2}{*}{$\sum^{\infty}$} \\
\hline & & {$[\mathrm{cm}]$} & & & {$[\%]$} & \multicolumn{2}{|c|}{$\left[g^{\cdot} \mathrm{kg}^{-1}\right]$} & & \multicolumn{3}{|c|}{$\left[\mathrm{cmol}(+) \cdot \mathrm{kg}^{-1}\right]$} & & \multicolumn{2}{|c|}{$\left[\mathrm{mg} \cdot 100 \mathrm{~g}^{-1}\right]$} & \\
\hline \multicolumn{16}{|c|}{ Results of analyses made in 1978} \\
\hline \multirow[t]{4}{*}{1} & Ap & $0-15$ & 7.12 & 6.11 & 0.17 & 17.9 & 0.89 & 20.0 & 8.02 & 13.2 & 21.22 & 62.21 & 5.05 & 6.0 & 3.70 \\
\hline & Et & $15-45$ & 7.21 & 5.91 & 0.08 & 11.2 & 0.23 & 48.0 & 2.31 & 7.1 & 9.4 & 75.53 & 0.20 & 5.0 & 9.75 \\
\hline & $\mathrm{Bt}$ & $45-60$ & 6.91 & 5.99 & 0.12 & 0.88 & 0.11 & 8.0 & 3.12 & 11.4 & 14.4 & 78.2 & 0.10 & 3.0 & 7.15 \\
\hline & $\mathrm{BC}$ & $60-120$ & 6.88 & 5.89 & 0.0 & - & - & - & 3.70 & 7.10 & 10.8 & 65.7 & 0.12 & 2.2 & 5.92 \\
\hline \multirow[t]{4}{*}{2} & Ap & 0-17 & 7.18 & 7.00 & 0.12 & 30.20 & 0.91 & 33.0 & 2.90 & 10.7 & 13.6 & 78.68 & 5.52 & 5.0 & 2.00 \\
\hline & $\mathrm{AC}$ & $17-30$ & 5.67 & 4.71 & 0.0 & 19.00 & 0.42 & 45.0 & 1.11 & 9.6 & 10.7 & 89.72 & 1.00 & 1.50 & 5.95 \\
\hline & $2 \mathrm{Cg}$ & $30-50$ & 5.91 & 5.98 & 0.0 & 1.86 & 0.09 & 20.7 & 0.80 & 14.8 & 15.6 & 94.87 & 0.30 & 2.50 & 3.25 \\
\hline & $2 \mathrm{G}$ & $50-70$ & 5.78 & 5.61 & 0.0 & - & - & - & 1.30 & 7.3 & 8.6 & 84.90 & 0.10 & 2.15 & 2.50 \\
\hline
\end{tabular}


Table 4. Cont.

\begin{tabular}{|c|c|c|c|c|c|c|c|c|c|c|c|c|c|c|c|}
\hline \multirow{2}{*}{$\stackrel{0}{0}$} & \multirow{2}{*}{ 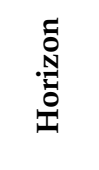 } & مَ & : & $\underset{ت}{ت}$ & $0_{0}^{\infty}$ & فํํㄹ & 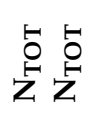 & \multirow[t]{2}{*}{$\underset{ن}{Z}$} & 焉 & $\omega$ & $\mapsto \underset{U}{U}$ & \multirow{2}{*}{$\begin{array}{l}>\infty \\
{[\%]}\end{array}$} & $0_{n}^{n}$ & O & \multirow{2}{*}{$\sum_{1}^{\infty 0}$} \\
\hline & & {$[\mathrm{cm}]$} & & & {$[\%]$} & \multicolumn{2}{|c|}{$\left[\mathrm{g} \cdot \mathrm{kg}^{-1}\right]$} & & \multicolumn{3}{|c|}{$\left[\mathrm{cmol}(+) \cdot \mathrm{kg}^{-1}\right]$} & & \multicolumn{2}{|c|}{$\left[\mathrm{mg} \cdot 100 \mathrm{~g}^{-1}\right]$} & \\
\hline \multirow[t]{4}{*}{3} & Ap & $0-12$ & 7.21 & 6.51 & 1.10 & 20.71 & 0.87 & 24.0 & 21.72 & 26.7 & 48.4 & 55.17 & 13.00 & 10.0 & 5.10 \\
\hline & Ees & $12-28$ & 6.51 & 5.89 & 0.0 & 0.51 & 0.53 & 1.0 & 2.15 & 5.12 & 7.27 & 70.43 & 0.70 & 0.50 & 2.12 \\
\hline & Bhs & $28-48$ & 5.21 & 4.50 & 0.0 & 0.32 & 0.04 & 8.0 & 4.57 & 1.12 & 5.69 & 19.68 & 0.10 & 0.50 & 4.22 \\
\hline & $\mathrm{C}$ & $48-110$ & 5.19 & 4.35 & 0.0 & - & - & - & 5.14 & 0.75 & 5.89 & 13.10 & 0.10 & 0.31 & 2.10 \\
\hline \multirow[t]{3}{*}{4} & $\mathrm{Au}$ & $0-16$ & 6.51 & 6.11 & 0.0 & 49.00 & 0.89 & 55.0 & 10.60 & 44.3 & 54.9 & 80.69 & 2.40 & 5.00 & 7.40 \\
\hline & $\mathrm{AC}$ & $16-55$ & 6.05 & 6.07 & 0.0 & 2.12 & 0.13 & 16.3 & 2.81 & 21.2 & 24.01 & 88.30 & 0.70 & 4.00 & 6.20 \\
\hline & $\mathrm{Cg}$ & 55-110 & 6.00 & 5.77 & 0.0 & - & - & - & 2.10 & 16.2 & 18.3 & 88.50 & 0.55 & 3.30 & 4.10 \\
\hline \multirow[t]{3}{*}{5} & Ap & $0-10$ & 8.02 & 7.30 & 15.89 & 26.43 & 1.14 & 23.0 & 2.66 & 66.6 & 69.26 & 96.16 & 0.41 & 5.0 & 3.95 \\
\hline & $\mathrm{ACca}$ & $10-21$ & 8.66 & 7.41 & 13.6 & 1.13 & 0.34 & 3.3 & 0.96 & 38.3 & 39.26 & 97.55 & 0.51 & 5.5 & 4.10 \\
\hline & Rca & $21-35$ & 8.78 & 7.71 & - & - & - & - & - & - & - & - & - & - & - \\
\hline \multicolumn{16}{|c|}{ Results of analyses made in 2005} \\
\hline \multirow[t]{4}{*}{1} & Ap & $0-15$ & 7.66 & 6.97 & 2.78 & 12.40 & 1.09 & 11.3 & 3.12 & 21.21 & 24.33 & 87.18 & 3.55 & 8.30 & 5.0 \\
\hline & Et & $15-45$ & 8.02 & 6.55 & 1.39 & 9.70 & 0.35 & 27.7 & 0.98 & 10.09 & 11.07 & 91.15 & 0.10 & 6.60 & 12.0 \\
\hline & $\mathrm{Bt}$ & $45-60$ & 7.03 & 6.41 & 1.98 & 0.53 & 0.02 & 26.5 & 2.11 & 15.90 & 18.01 & 88.28 & 0.10 & 4.20 & 9.67 \\
\hline & $\mathrm{BC}$ & $60-120$ & 7.00 & 6.03 & 0.03 & & & & 2.96 & 8.41 & 11.37 & 73.97 & - & 3.0 & 7.80 \\
\hline \multirow[t]{4}{*}{2} & Ap & $0-17$ & 7.65 & 7.44 & 1.12 & 24.92 & 1.07 & 23.2 & 2.00 & 13.81 & 15.81 & 87.35 & 5.30 & 5.55 & 2.11 \\
\hline & $\mathrm{AC}$ & $17-30$ & 6.27 & 6.05 & 0.03 & 20.52 & 0.28 & 73.2 & 0.70 & 10.77 & 11.47 & 93.90 & 0.85 & 2.03 & 6.4 \\
\hline & $2 \mathrm{Cg}$ & $30-50$ & 6.12 & 6.02 & - & 1.33 & 0.07 & 19.0 & 0.45 & 16.11 & 16.56 & 97.28 & 0.25 & 3.31 & 4.0 \\
\hline & $2 \mathrm{G}$ & $50-70$ & 6.03 & 6.00 & - & - & - & & 1.10 & 8.09 & 9.19 & 88.03 & 0.13 & 2.27 & 3.02 \\
\hline \multirow[t]{4}{*}{3} & Ap & $0-12$ & 7.87 & 7.58 & 3.79 & 11.53 & 0.98 & 11.7 & 1.71 & 36.56 & 38.27 & 95.53 & 7.18 & 14.7 & 9.11 \\
\hline & Ees & $12-28$ & 7.22 & 7.03 & 23.42 & 0.32 & 0.09 & 3.6 & 0.64 & 10.24 & 10.88 & 94.12 & 0.55 & 1.10 & 4.23 \\
\hline & Bhs & $28-48$ & 7.11 & 7.09 & 2.47 & 0.09 & 0.05 & 1.8 & 0.23 & 1.91 & 2.14 & 89.25 & 0.05 & 0.90 & 5.9 \\
\hline & $\mathrm{C}$ & $48-110$ & 6.32 & 5.96 & 0.18 & - & - & & 1.12 & 0.94 & 2.06 & 45.63 & - & 0.42 & 2.70 \\
\hline \multirow[t]{3}{*}{4} & $\mathrm{Au}$ & $0-16$ & 7.04 & 6.89 & 1.08 & 46.13 & 0.97 & 47.5 & 7.33 & 46.21 & 53.54 & 86.31 & 2.10 & 6.11 & 8.56 \\
\hline & $\mathrm{AC}$ & $16-55$ & 6.91 & 6.77 & 0.03 & 20.04 & 0.42 & 47.7 & 1.79 & 27.90 & 29.69 & 93.97 & 0.34 & 4.89 & 7.0 \\
\hline & $\mathrm{Cg}$ & 55-110 & 6.44 & 6.12 & - & - & - & & 1.55 & 18.66 & 20.21 & 92.33 & 0.30 & 4.00 & 4.30 \\
\hline 5 & Ap & $0-10$ & 8.44 & 8.01 & 17.62 & 22.20 & 1.13 & 19.6 & 1.99 & 71.8 & 73.79 & 97.30 & 0.36 & 6.11 & 4.11 \\
\hline & $\mathrm{ACca}$ & $10-21$ & 8.70 & 7.55 & 14.62 & 1.29 & 0.37 & 3.5 & 0.78 & 40.0 & 40.78 & 98.09 & 0.45 & 7.23 & 5.34 \\
\hline & Rca & $21-35$ & 8.88 & 7.80 & - & - & - & - & - & - & - & - & - & - & - \\
\hline Resu & of an & es mad & 2019 & & & & & & & & & & & & \\
\hline 1 & Ap & $0-15$ & 7.70 & 6.80 & 2.66 & 11.30 & 1.12 & 10.1 & 3.02 & 21.21 & 24.23 & 87.54 & 3.15 & 8.79 & 5.23 \\
\hline & Et & $15-45$ & 7.92 & 6.50 & 1.20 & 9.10 & 0.38 & 23.9 & 0.92 & 10.09 & 11.01 & 91.64 & 0.08 & 7.09 & 12.93 \\
\hline & $\mathrm{Bt}$ & $45-60$ & 7.13 & 6.49 & 1.55 & 0.43 & 0.23 & 1.8 & 2.03 & 15.90 & 17.93 & 88.68 & 0.10 & 4.54 & 10.07 \\
\hline & $\mathrm{BC}$ & $60-120$ & 7.20 & 6.55 & 0.05 & & & & 2.16 & 8.41 & 10.57 & 79.56 & - & 3.70 & 7.62 \\
\hline 2 & Ap & $0-17$ & 7.50 & 7.00 & 1.05 & 21.22 & 1.09 & 19.4 & 2.12 & 13.81 & 15.93 & 86.69 & 4.92 & 5.61 & 2.77 \\
\hline & $\mathrm{AC}$ & $17-30$ & 6.40 & 6.25 & 0.05 & 18.61 & 0.25 & 74.5 & 0.56 & 10.77 & 11.33 & 95.06 & 0.75 & 2.45 & 6.92 \\
\hline & $2 \mathrm{Cg}$ & $30-50$ & 6.00 & 5.89 & - & 1.93 & 0.05 & 38.6 & 0.38 & 16.11 & 16.49 & 97.70 & 0.27 & 3.91 & 4.88 \\
\hline & $2 \mathrm{G}$ & $50-70$ & 6.05 & 5.97 & - & - & - & - & 1.70 & 8.09 & 9.79 & 82.64 & 0.11 & 2.59 & 3.00 \\
\hline 3 & Ap & $0-12$ & 7.90 & 7.65 & 3.99 & 10.43 & 1.01 & 10.3 & 1.55 & 36.56 & 38.11 & 95.93 & 7.02 & 15.2 & 10.67 \\
\hline & Ees & $12-28$ & 7.31 & 7.12 & 24.11 & 0.29 & 0.10 & 2.9 & 0.54 & 10.24 & 10.78 & 94.99 & 0.38 & 1.43 & 5.12 \\
\hline & Bhs & $28-48$ & 7.00 & 6.83 & 2.01 & 0.07 & 0.04 & 1.7 & 0.27 & 1.91 & 2.18 & 87.61 & 0.25 & 1.23 & 5.20 \\
\hline & $\mathrm{C}$ & $48-110$ & 6.42 & 6.06 & 0.20 & - & - & - & 0.98 & 0.94 & 1.92 & 48.96 & - & 0.61 & 2.34 \\
\hline 4 & $\mathrm{Au}$ & $0-16$ & 7.12 & 6.90 & 0.98 & 46.91 & 1.02 & 45.9 & 6.43 & 46.21 & 52.64 & 87.78 & 2.21 & 7.08 & 8.99 \\
\hline & $\mathrm{AC}$ & $16-55$ & 6.51 & 6.33 & 0.03 & 19.84 & 0.82 & 24.2 & 1.29 & 27.90 & 29.19 & 95.58 & 0.27 & 5.24 & 7.69 \\
\hline & $\mathrm{Cg}$ & 55-110 & 6.13 & 6.00 & - & - & - & - & 1.22 & 18.66 & 19.88 & 93.86 & 0.36 & 4.74 & 4.90 \\
\hline 5 & Ap & $0-10$ & 8.51 & 8.21 & 18.07 & 21.30 & 1.19 & 17.8 & 1.59 & 71.8 & 73.39 & 97.83 & 0.31 & 6.99 & 4.17 \\
\hline & $\mathrm{ACca}$ & $10-21$ & 8.72 & 7.45 & 13.11 & 1.21 & 0.36 & 3.3 & 0.58 & 40.0 & 40.58 & 98.57 & 0.40 & 7.04 & 5.07 \\
\hline & Rca & 21-35 & 8.90 & 7.81 & - & - & - & - & - & - & - & - & - & - & - \\
\hline
\end{tabular}

The $\mathrm{pH}_{\mathrm{KCl}}$ from 5.50 to 7.20 is considered the optimum range for biological processes related to metabolism of majority of plants and soil microorganisms. It can be assumed that the analysed soils showed many features of limed soils, which were particularly apparent in the podsolic and fallow soils (Profiles 1 and 3). The results of research conducted in 
1978-2005 revealed great changes in $\mathrm{pH}$ (Figure 3). Since 2005, changes in $\mathrm{pH}$ of humus horizon have been slight, with a maximum change of 0.2 units in profiles no. 3,4 and 5 .

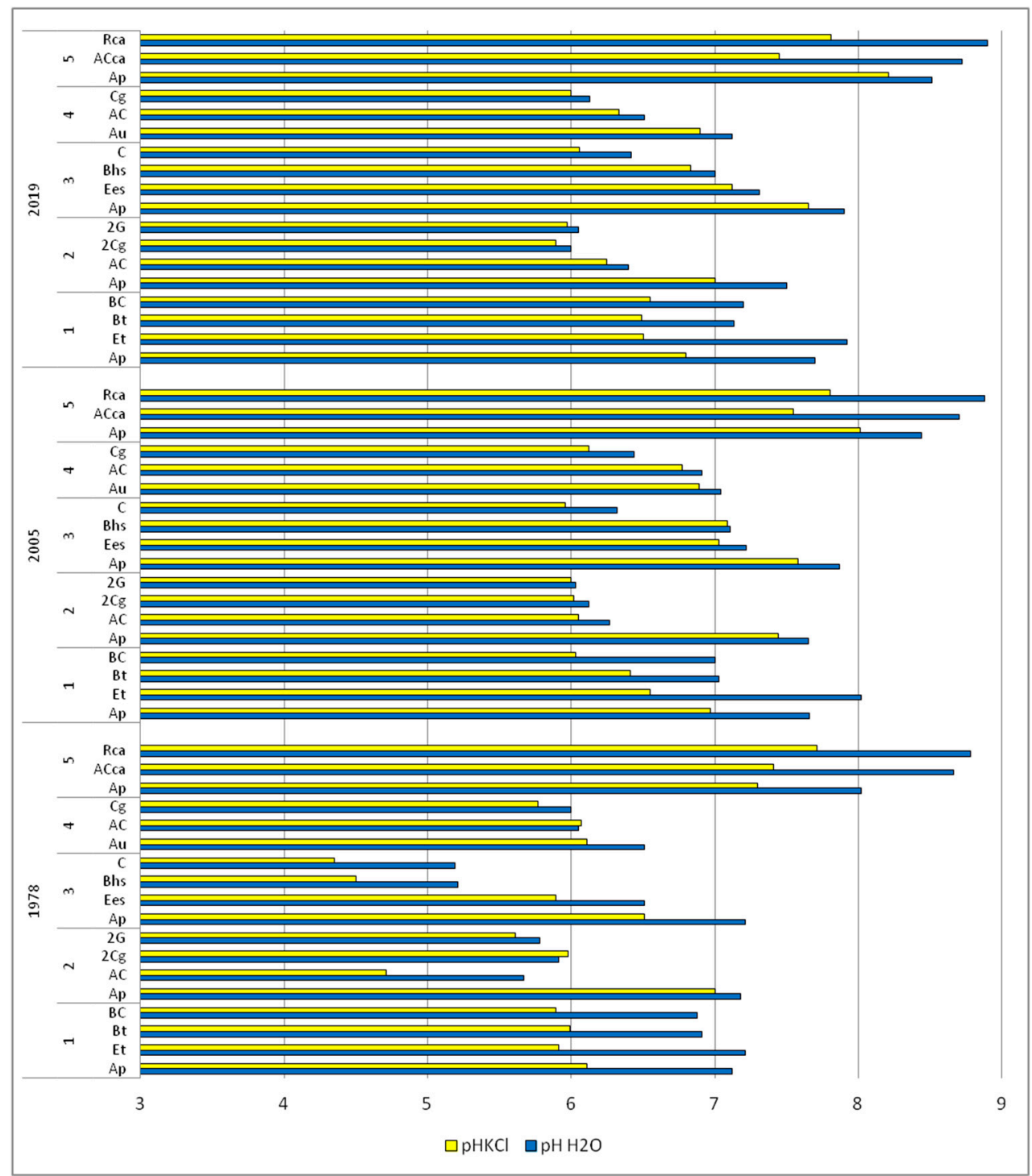

Figure 3. Changes in $\mathrm{pH}_{\mathrm{H} 2 \mathrm{O}}$ and $\mathrm{pH}_{\mathrm{KCl}}$ value ranges in individual horizons of the analysed soils between 1978 and 2019.

In the analysed profiles, the neutral and alkaline reaction was maintained at the topsoil horizons and decreased at level of the parent rock regardless of its type (Table 4, Figure 4).

Calcium carbonate content in the soil profiles has changed since 1978. At the beginning of the research, it was present only in the surface soil horizons $\left(0.08 \%\right.$ to $\left.1.1 \% \mathrm{CaCO}_{3}\right)$. The highest value of calcium was revealed in the profile no. 5 (Rendzic leptosol), where the value partly depended on the nature of the bedrock (Devonian limestones). Until 2005, carbonates also appeared in those horizons where it had not been present before. In 2005, carbonate content was reported (0.03-23.42\%). The highest increase of $\mathrm{CaCO}_{3}$ was found in the profile no. 3 (Haplic Podzol). The research carried out 14 years later showed a slow decrease in the carbonate content, with the exception of the Haplic Podzol formed out of sands with a low sorption capacity and in the humus horizon of the profile no. 5 (Figure 5). 


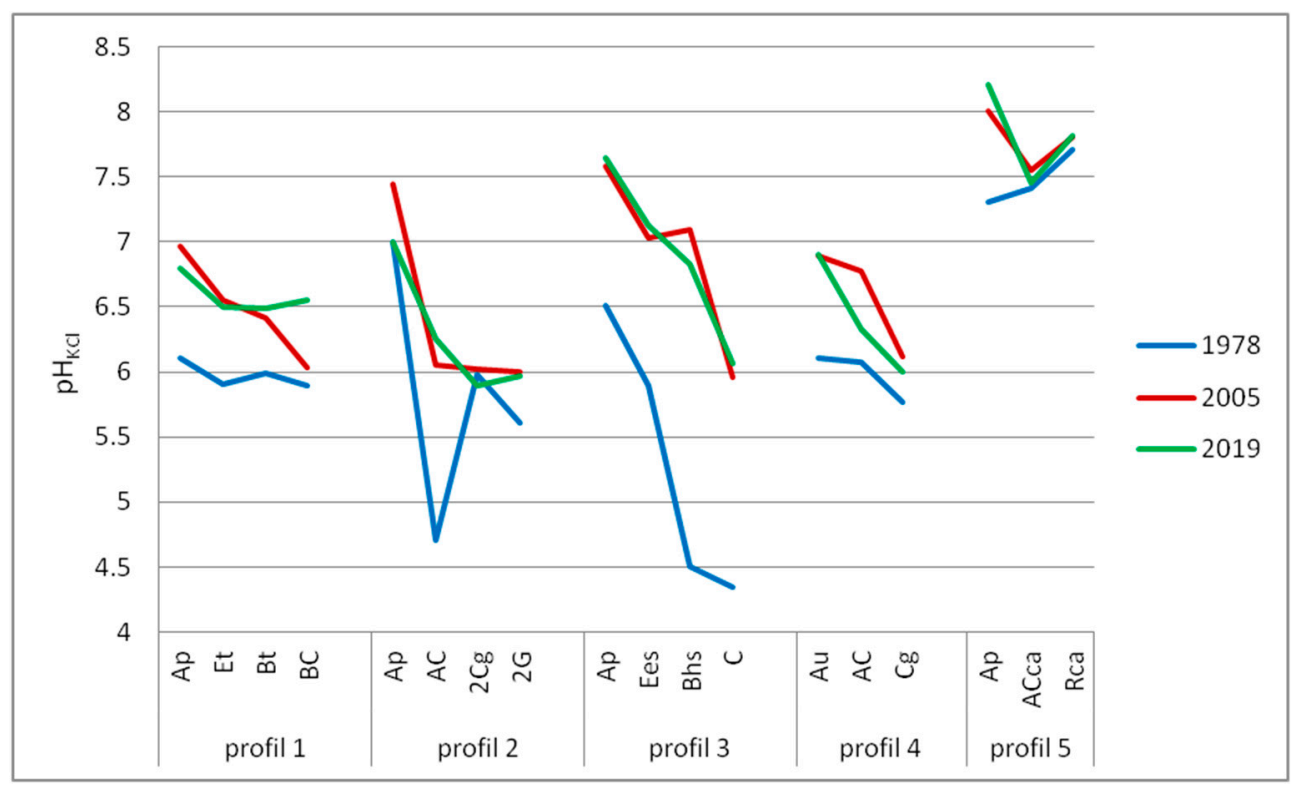

Figure 4. Dynamics of $\mathrm{pH}_{\mathrm{KCl}}$ value ranges in individual horizons of the analysed soils between 1978 and 2019.

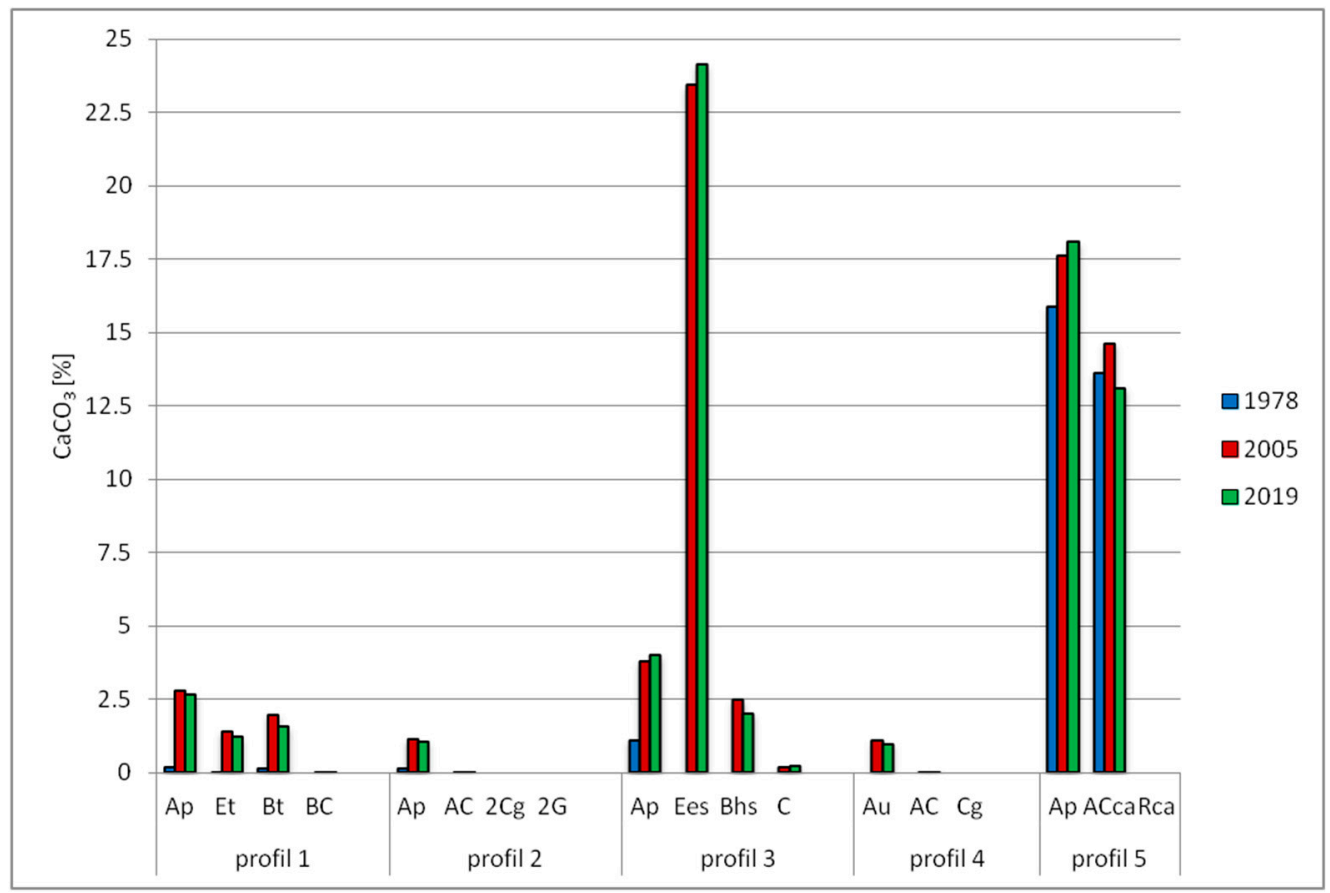

Figure 5. Dynamics of $\mathrm{CaCO}_{3}(\%)$ content in individual horizons of the analysed soils between 1978 and 2019.

In the analysed soil profiles, the content of $\mathrm{C}_{\text {org }}$ in humus horizons ranged from $179.1 \mathrm{~g} \cdot \mathrm{kg}^{-1}$ to $998.0 \mathrm{~g} \cdot \mathrm{kg}^{-1}$ in 1978 . For 40 years, the low decline of $\mathrm{C}_{\text {org }}$ took place, and in 2019 , its content in the analysed soil profiles ranged from $104.3 \mathrm{~g} \cdot \mathrm{kg}^{-1}$ to $869.1 \mathrm{~g} \cdot \mathrm{kg}^{-1}$ (Table 4, Figure 6). The most significant changes in $\mathrm{C}_{\text {org }}$ were found in Mollic gleysol (no. 4). 


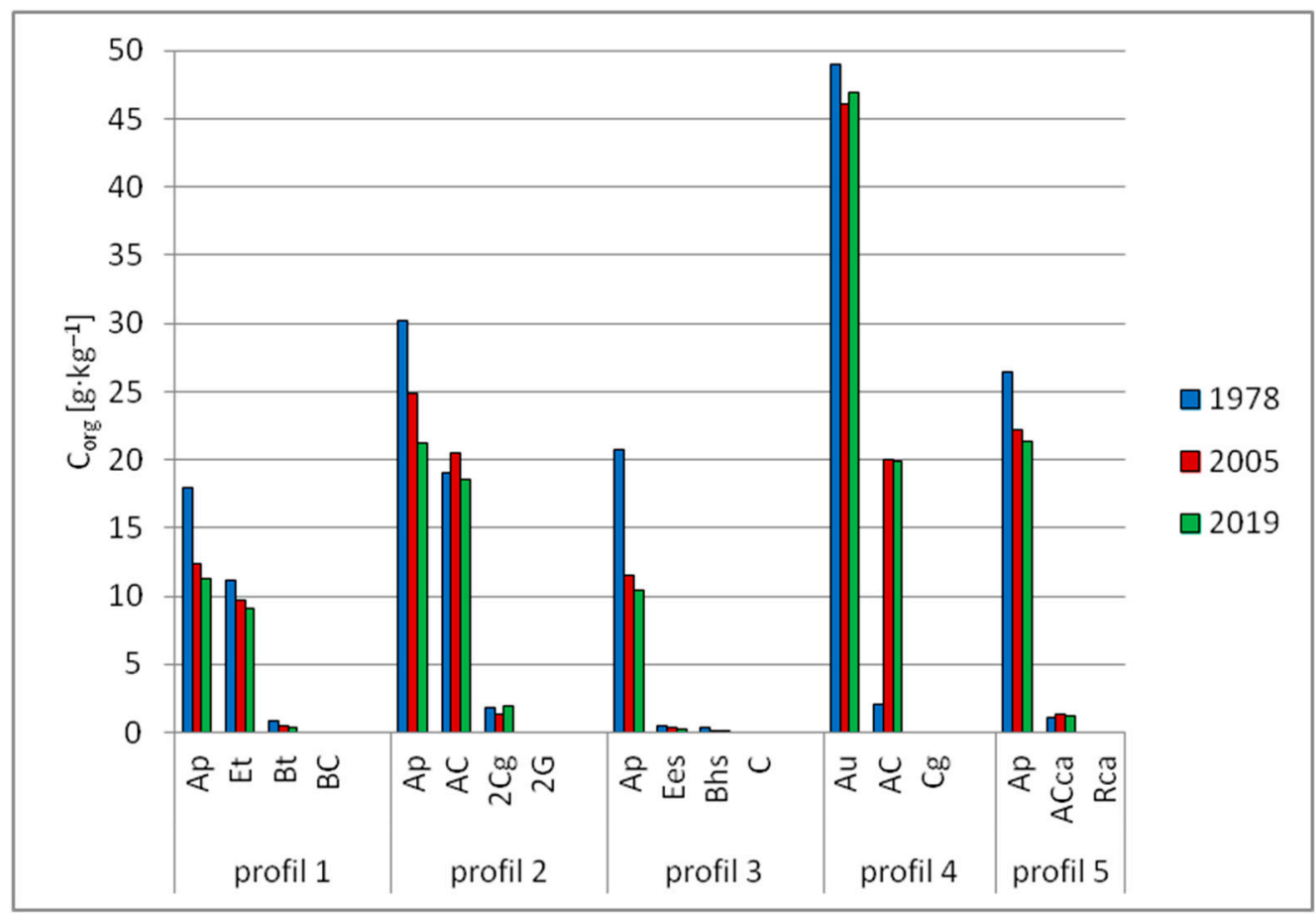

Figure 6. Dynamics of $C_{\text {org }}\left(\mathrm{g} \cdot \mathrm{kg}^{-1}\right)$ content in individual horizons of the analysed soils between 1978 and 2019.

Nitrogen in soils belongs to active and dynamic elements. Its content in the surface horizon of soils in Poland has been estimated to be from $0.02 \%$ to $0.035 \%$ and depends on a number of factors, including the climate ones [33-35].

In the analysed profiles, the highest level of nitrogen was observed in humus, and its content decreased with depth. During the period from 1978 to 2019, the nitrogen content underwent minor changes, with a slight growing tendency in all analysed soil horizons (Table 4).

Phosphorus and potassium are essential elements of yields enrichment. Their content in the soil is one of the most important factors determining soil fertility. Overall content of phosphorus in the arable horizon of soil depends on the type of bedrock, and the main secondary source is dead organism remains [36]. The most mobile phosphorus (digestible) is found in soils with a slightly acid reaction.

In the analysed soils, the phosphorus level took the highest values in the surface horizons (Figure 7). The content of phosphorus in most analysed soils started to decline in 1978. This tendency was most strongly evident in the humus horizon of the Haplic Podzol, with the simultaneous increase in the phosphorus content in the illuvial horizon of that soil. Declines in the content of available phosphorus are caused by the high soil $\mathrm{pH}$, which causes the re-establishment of this element [37].

Similarly to the potassium content, the magnesium content was also higher in the analysed soils over the last 40 years, with the strongest dynamics in the Haplic Podzol.

The sorption complex of analysed soils was characterised by high saturation with alkaline cations. The level of humus saturation showed a rising tendency: From 55.17\% in 1978 to $86.1 \%$ in 2005 and $86.69 \%$ in 2019 (Table 4). At present, this value exceeds $85 \%$ in most of the horizons. 


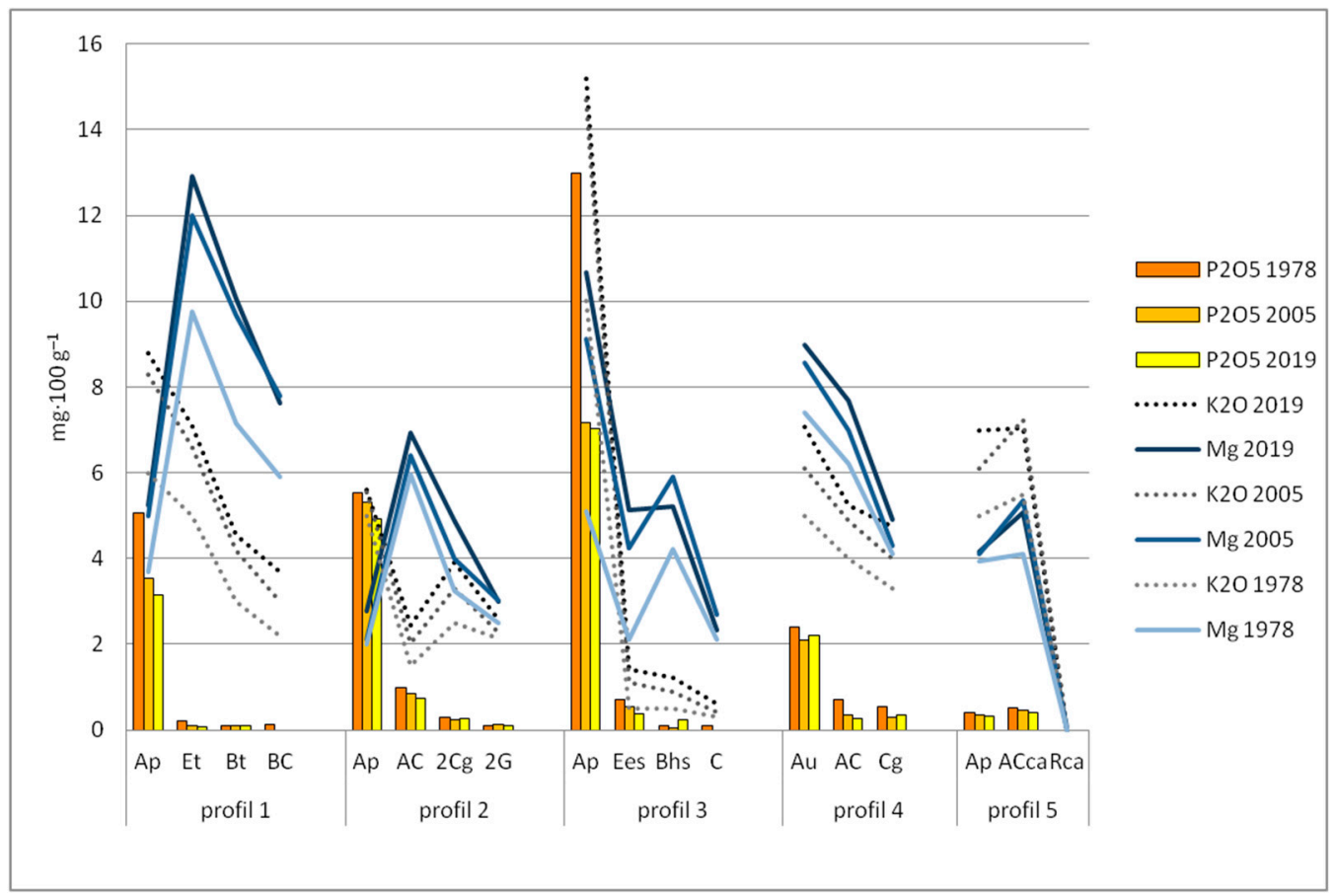

Figure 7. Changes in the content of digestible components $\left(\mathrm{P}_{2} \mathrm{O}_{5}, \mathrm{~K}_{2} \mathrm{O}, \mathrm{Mg}\left(\mathrm{mg} \cdot 100 \mathrm{~g}^{-1}\right)\right)$ in various horizons of the analysed soils in 1978-2019.

\section{Discussion}

The cement and lime industry plays an important role in the world's economy. However, despite its popularity and profitability, the use of more and more pro-ecological technologies, improvement of production processes and use of alternative fuels, the cement and lime production consequently leads, inter alia, to environmental pollution through increased alkalisation of habitats [8]. Therefore, the cement industry faces many challenges related to environmental protection and sustainability issues [11].

Acid rocks carried by glaciers from Scandinavia compose $90 \%$ of Poland's soils, which are inherently poor in alkaline cations [38]. In the years 1957-1965, i.e., before the opening of "Nowiny" Cement Plant, acidic soils accounted for as much as $67 \%$ of the area of the Sitkówka-Nowiny commune (67\%), while in $2008,94 \%$ of the soils of this commune were classified as alkaline soils [23].

The most constant and least susceptible to anthropopressure was the Haplic Podzol soil species, which results from its granulometric composition. It has a significant importance for many physical and chemical properties of soils, including $\mathrm{pH}$, the natural content of contaminants in the soil and the sorption capacity of soils, directly influencing the processes of contaminants migration in the environment. The research shows that the granulometric composition of soil has not changed, nor has its general morphological structure.

The most frequently mentioned changes observed in soils under the influence of alkalization are increases in $\mathrm{pH}$ values, increases in carbonate content and increases in buffer volumes $[6,16,39]$. Similar patterns were observed in the analysed soil profiles.

The influence of soil $\mathrm{pH}$ on the phosphorus and nitrogen content has been analysed by various researchers [40]. It was proven that the abovementioned influence differed regarding these two elements. In contrast to nitrogen, acidity reduction and the enrichment of soil in calcium (as a result of liming) reduced the releasing of mineral phosphorus in the soil [41]. Soil reaction is pointed as a decisive factor of phosphorus bioavailability and the possibility of its migration into the soil profile depth [42]. With soil $\mathrm{pH}$ higher than 
6.5, phosphorus is immobilised by free $\mathrm{Fe}$ and $\mathrm{Al}$ oxides, while in the case of alkaline, the reaction by calcium ions creates sparingly soluble salts $[43,44]$.

In contrast to phosphorus, potassium is almost completely bound to the mineral part of soils. Total potassium content in arable soils is related to mineral soil composition and content of colloidal and dusty elements [45]. Therefore, higher contents of this element are found in heavier soils with a high-capacity sorption complex. When it comes to light soils with a small capacity sorption complex, there is a greater loss of this element as a result of leaching by atmospheric precipitation $[4,46]$. The potassium content of studied soils has been increasing since 1977. The highest values are found in surface soils, while the highest changes in the content of this element are observed in the Haplic Podzol.

Another issue related to habitat alkalization is the change in organic matter content. Soil organic matter is the basic indicator of soil quality determining its physicochemical characteristics, such as sorption, buffer capacity and biological processes, which determine many changes, referred to as biological activity. High humus content in soils is a factor stabilizing their structure, reducing their susceptibility to compaction and degradation due to water and wind erosion. The preservation of soil humus resources is important not only for maintaining the productive functions of soils, but also for the role of soils in carbon sequestration from the atmosphere [47].

In many cases, a variety of human impacts which affect the nature result in the loss of soil organic carbon. The use of land for grassland is indicated as the friendliest for soil organic carbon preservation [48].

Over many years of research, a clear loss of carbon (soil humus) was observed. This condition was caused not only by the long-term immission of cement and lime dusts into the soil but also by other factors. These include the change of land use (in 1978, the analysed soils were used for agricultural purposes, mainly rye was grown on them and some were used as hay meadows). Since 1990, the soils have not been used for agricultural purposes. Nowadays, it is a green area (fallow fields subject to natural succession or meadows with mowing remaining on the site). The change of the land use has also been affected by climatic changes (over the observed 40 years, the average annual temperature increased by $1.5-1.8^{\circ} \mathrm{C}$ according to the Meteorological Station in Suków), which is conducive to the decrease of humus resources. This fact was pointed out by Antil and Singh (2007), Zimmer et al. (2005) and Elmer et al. (2000) [49-51]. In addition, the presence of $\mathrm{CaCO}_{3}$ may affect the temperature of humus levels. This thesis was confirmed by Zimmer et al. (2005) and Elmer, Baumecker (2008), where a subsequent increase in the temperature of humus levels by $0.02{ }^{\circ} \mathrm{C}$ was followed by a loss of $\mathrm{C}_{\text {org }}$ by $15-20 \%$ (depending on soil type) [50,52]. Trends related to general climate warming are an undisputed fact and should also be related to general organic matter resources.

It seems that the observed soil humus loss should be related to a smaller illuvium, the displacement of humus compounds deep into the profiles and the higher anthropogenic chemical and biological activity of soils. This has been confirmed by long-term studies conducted under conditions of boron habitat alkalization [16] and model studies [52,53]. These studies have shown that alkaline conditions (higher $\mathrm{pH}$ values) are conducive to a decrease in the total content of organic matter in the soil, because under alkaline conditions, the organic matter is pepticized to a much greater extent due to an increase in surface charge. Another reason for lowering the carbon resources may be a change in hygrothermal conditions, such as droughts and higher air temperatures during the period of intensive plant growth, which also influence the decomposition rate [54].

\section{Conclusions}

Over 50 years since launching, the Dyckerhoff Polska Sp. z o.o. Nowiny Cement Plant and other cement and lime plants have caused great modifications in soil cover functioning in this area. In spite of dust emission and immission decreasing, the effects of cement plant operations are still visible, especially in the Haplic Podzol, with low buffer capacities. The regeneration of soils and return to the original properties are hampered by 
the noticeable drop of alkaline dusts of $>33.0 \mathrm{~g} / \mathrm{m}^{2} /$ year and other anthropogenic factors (dense network of roads, fertilisation).

Constant immission of cement and lime dust has had a significant influence on the chemical quality and basic properties of the soils since 1966. It was found that the long-term effect of alkaline immission resulted in:

- Higher $\mathrm{pH}$ of soil, and the highest values $\mathrm{pH}_{\mathrm{KCl}}$ in humus horizons;

- Increased $\mathrm{CaCO}_{3}$ content in organic and organic-mineral horizons;

- Decreased $C_{\text {org }}$ content, especially in humus horizons. The trends of these changes were multifaceted and were also related to the change of land use and increase of average annual air temperatures; and

- A raised level of saturation of sorption complex with alkaline cations, despite reducing dust immission to the soils.

In recent years, the dynamics of observed changes have been getting smaller, and this is proven in the research results.

Author Contributions: Conceptualization, A.Ś.; methodology, A.Ś.; A.G.; I.T.-W.; software, A.Ś.; A.G.; I.T.-W.; validation, A.Ś.; A.G.; I.T.-W.; formal analysis, A.Ś.; A.G.; I.T.-W.; investigation, A.Ś.; A.G.; I.T.-W.; resources, A.Ś.; A.G.; I.T.-W.; data curation, A.Ś.; A.G.; I.T.-W.; writing—original draft preparation, A.S.; A.G.; I.T.-W.; writing—review and editing, A.S.; A.G.; I.T.-W.; visualization, A.G.; I.T.-W.; supervision, A.Ś.; project administration, A.Ś.; funding acquisition, A.Ś. All authors have read and agreed to the published version of the manuscript.

Funding: This research received no external funding.

Institutional Review Board Statement: The study did not require ethical approval.

Informed Consent Statement: Not applicable.

Acknowledgments: We thank the Department of Agriculture and Rural Development of the Marshal's Office of the Świętokrzyskie Voivodeship_for supporting this publication.

Conflicts of Interest: The authors declare no conflict of interest.

\section{References}

1. Vogel, H.; Eberhardt, E.; Franko, U.; Lang, B.; Ließ, M.; Weller, U.; Wiesmeier, M.; Wollschläger, U. Quantitative Evaluation of Soil Functions: Potential and State. Front. Environ. Sci. 2019, 7, 164. [CrossRef]

2. Smreczak, B.; Ukalska-Jaruga, A.; Łysiak, M.; Strzelecka, J.; Niedźwiecki, J.; Sobich, D. Funkcje, jakość i usługi ekosystemowe gleb. Studia Rap. IUNG-PIB 2017, 54, 9-23.

3. Brevik, E.C.; Cerdà, A.; Mataix-Solera, J.; Pereg, L.; Quinton, J.N.; Six, J.; van Oost, K. The interdisciplinary nature of soil. Soil 2015, 1, 117-129. [CrossRef]

4. Instytut Uprawy Nawożenia i Gleboznawstwa-Państwowy Instytut Badawczy w Puławach. Monitoring Chemizmu Gleb Ornych w Polsce w Latach 2015-2017; Instytut Uprawy Nawożenia i Gleboznawstwa: Puławy, Poland, 2017.

5. Stepnowski, P.; Synak, E.; Szafranek, B.; Kaczyński, Z. Monitoring i Analityka Zanieczyszczeń w Środowisku; Wydawnictwo Uniwersytetu Gdańskiego: Gdańsk, Poland, 2010.

6. Sowunmi, K.; Shoga, S.; Adewumi, O.M.; Oriyomi, F.A.; Sowunmi, L. The Physiological and Microbiological Characteristics of Cement Dust Polluted Soil Around Lafarge Cement Industry. Res. Sq. 2020. [CrossRef]

7. Shah, K.; An, N.; Ma, W.; Ara, G.; Ali, K.; Kamanova, S.; Zuo, X.; Han, M.; Ren, X.; Xing, L. Chronic cement dust load induce novel damages in foliage and buds of Malus domestica. Sci. Rep. 2020, 10, 12186. [CrossRef]

8. Bilen, S.; Bilen, M.; Turan, V. Relationships between Cement Dust Emissions and Soil Properties. Pol. J. Environ. Stud. 2019, 28, 3089-3098. [CrossRef]

9. Zeb, K.; Ali, Y.; Khan, M.W. Factors influencing environment and human health by cement industry: Pakistan a case in point. Manag. Environ. Qual. Int. J. 2019, 30, 751-767. [CrossRef]

10. Amani, J.; Babu, G.K.; Lakshmipathi, R.; Rao, G.R.; Chandrasekhar, K. Effect of Cement Dust Deposition on Soil Microbial Properties. Int. J. Curr. Microbiol. App. Sci. 2018. [CrossRef]

11. Devi, K.S.; Lakshmi, V.V.; Alakanandana, A. Impacts of cement industry on environment-An overview. Asia Pac. J. Res. 2017, I, $156-161$.

12. Stanley, H.O.; Odu, N.N.; Immanuel, O.M. Impact of cement dust pollution on physicochemical and microbiological properties of soil around Lafarge cement WAPCO, EWEKORO, southwestern Nigeria. Int. J. Adv. Biol. Res. 2014, 4, 400-404.

13. Malczyk, P.; Rydlewska, M. Properties of soils surrounded Trzuskawica lime plant industry S.A., Department of Kujawy. Ecol. Chem. Eng. 2011, 18, 9-10. 
14. Kumar, S.; Singh, N.; Kumar, V.; Sunisha, B.; Preeti, S.; Deepali, D.; Nath, S. Impact of dust emission on plant vegetation in the vicinity of cement plant. Environ. Eng. Manag. J. 2008, 7, 31-35. [CrossRef]

15. Ivanov, Y.V.; Kartashov, A.V.; Ivanova, A.I.; Ivanov, V.P.; Marchenko, S.I.; Nartov, D.I.; Kuznetsov, V.V. Long-term impact of cement plant emissions on the elemental composition of both soils and pine stands and on the formation of Scots pine seeds. Environ. Pollut. 2018, 243, 1383-1393. [CrossRef] [PubMed]

16. Świercz, A. Analiza Procesów Glebowych i Przekształceń Roślinnych w Zalkalizowanych Siedliskach Leśnych Regionu Świętokrzyskiego; Komitet Człowiek i Środowisko" przy Prezydium PAN, Zesz. Nauk.: Warszawa, Polind, 2005; Volume 39, pp. 1-21.

17. Paal, J.; Degtjarenko, P.; Suija, A.; Liira, J. Vegetation responses to long-term alkaline cement dust pollution in Pinus sylvestrisdominated boreal forests-Niche breadth along the soil pH gradient. Appl. Veg. Sci. 2012, 16, 248-259. [CrossRef]

18. Jaworska, H.; Dąbrowska-Naskręt, H.; Sawilska, K. Wpływ pyłów cementowych na niektóre właściwości gleb oraz stan drzewostanów sosnowych w otoczeniu zakładów cementowo-wapienniczych "Lafarge" w Bielawach. Proc. ECOpole Towarzystwo Chemii i Inżynierii Ekologicznej 2010, 4, 141-146.

19. Drack, J.M.E.; Vázquez, D.P. Morphological response of a cactus to cement dust pollution. Ecotoxicol. Environ. Saf. 2018, 148, 571-577. [CrossRef]

20. Ots, K.; Mandre, M. Monitoring of heavy metals uptake and allocation in Pinus sylvestris organs in alkalised soil. Environ. Monit. Assess. 2012, 184, 4105-4117. [CrossRef]

21. Haapala, H.; Goltsova, N.; Lodenius, M. Heavy metal solubility in podzolic soils exposed to the alkalizing effect of air pollutants. Environ. Pollut. 2001, 115, 33-41. [CrossRef]

22. Annuka, E. The impact of emission from cement plant on forest landscape. Proceedings of the Estonian Academy of Sciences. Ecology 1994, 4, 118-127.

23. Strategia Rozwoju Gminy Sitkówka-Nowiny do 2015 r., Część I Raport o stanie Gminy; Świętokrzyskie Centrum Fundacji Rozwoju Demokracji Lokalnej: Kielce, Poland, 2008.

24. Lorenc, H.; Suwalska-Bogucka, M. Thermal tendencies of winters in Poland as the indicator of climate variability. Zesz. Nauk. UJ. Prace Geogr. 1996, 102, 365-374.

25. Lorenc, H. Termiczno-Opadowa Ocena Klimatycznych Sezonów Roku w Polsce oraz Tendencje Czasowo-Przestrzenne; Projekt badawczy M-9; IMGW: Warszawa, Poland, 2000.

26. Bednarek, R.; Dziadowiec, H.; Pokojska, U.; Prusinkiewicz, Z. Badania Ekologiczno-Gleboznawcze; PWN: Warszawa, Poland, 2005.

27. Kowalkowski, A.; Swałdek, M. Analiza Podstawowych Chemicznych Właściwości Gleb z Elementami Analityki; WSP: Kielce, Poland, 1991.

28. Karczewska, A.; Kabała, C. Metodyka Analiz Laboratoryjnych Gleb i Roślin; Uniwersytet Przyrodniczy we Wrocławiu: Wrocław, Poland, 2008.

29. Azimi, S.; Ludwig, A.; Thevenot, R.D.; Colin, J.-L. Trace metal determination in total atmospheric deposition in rural and urban areas. Sci. Total Environ. 2003, 308, 247-256. [CrossRef]

30. Świercz, A. Antropogeniczne zmiany właściwości chemicznych gleb powierzchniowych Obniżeń Chęcińskich. Roczniki Gleboznawcze 2007, 58, 141-150.

31. Systematyka Gleb Polski; Polskie Towarzystwo Gleboznawcze, Komisja Genezy, Klasyfikacji i Kartografii Gleb: Wrocław, Poland; Warszawa, Poland, 2019.

32. Persson, T.; Andersson, S.; Bergholm, J.; Grönqvist, T.; Högbom, L.; Vegerfors, B.; Wirén, A. Long-Term Impact of Liming on Soil $\mathrm{C}$ and $\mathrm{N}$ in a Fertile Spruce Forest Ecosystem. Ecosystems 2020. [CrossRef]

33. Watros, A.; Lipińska, H.; Lipiński, W.; Tkaczyk, P.; Krzyszczak, J.; Baranowski, P. Mineral nitrogen content in hydrographic areas of Poland depending on land use. Int. Agrophys. 2019, 33, 481-491. [CrossRef]

34. Harasim, A.; Igras, J.; Harasim, P. Zmiany zawartości azotu mineralnego w glebie mineralnej pod mieszanką pastwiskową w różnych stanowiskach. Pol. J. Agron. 2017, 30, 25-32.

35. Staszewski, Z. Azot w glebie i jego wpływ na środowisko. Zeszyty Naukowe Inżynieria Ladowa i Wodna w Kształtowaniu Środowiska 2011, 4, 50-58.

36. Jonczak, J.; Simansky, V.; Polláková, N. Zawartość i profilowe rozmieszczenie frakcji fosforu w uprawnych i leśnych czarnoziemach z poziomem cambic. Sylwan 2015, 159, 931-939.

37. Malczyk, P. Przewapnowanie gleb. Farmer 2013, 1, 1-3.

38. Hołubowicz-Kliza, G. Wapnowanie Gleb w Polsce; IUNG-PIB: Puławy, Poland, 2006.

39. Lamare, R.E.; Singh, O.P. Effect of cement dust on soil physico-chemical properties around cement plants in Jaintia Hills, Meghalaya. Environ. Eng. Res. 2020, 25, 409-417. [CrossRef]

40. Sapek, B.; Kalińska, D. Mineralizacja organicznych związków azotu w glebie w świetle długoletnich doświadczeń łąkowych IMUZ. Woda Środowisko Obszary Wiejskie 2004, 4, 183-200.

41. Sapek, B. Uwalnianie azotu i fosforu z materii organicznej gleby. Woda Środowisko Obszary Wiejskie 2010, 10, $229-256$.

42. Devau, N. Soil pH controls the environmental availability of phosphorus: Experimental and mechanistic modelling approaches. Appl. Geochem. 2009, 24, 2163-2174. [CrossRef]

43. Achat, D.L. Phosphorus status of soils from contrasting forested ecosystems in southwestern Siberia: Effects of microbiological and physicochemical properties. Biogeosciences 2013, 10, 733-752. [CrossRef] 
44. Darke, A.K.; Walbridge, M.R. Al and Fe biogeochemistry in a floodplain forest: Implications for P retention. Biogeochemistry 2000, 51, 1-32. [CrossRef]

45. Mercik, S. Chemia Rolna; Podstawy Teoretyczne i Praktyczne; SGGW: Warszawa, Poland, 2004.

46. Inspekcja Ochrony Środowiska. Monitoring Chemizmu Gleb Ornych w Polsce w Latach 2010-2012; Biblioteka Monitoringu Środowiska: Warszawa, Poland, 2012.

47. Stępień, W. Jak Przywracać Żyzność Gleb. Monografia Ochrona Bioróżnorodności Gleby Warunkiem Zdrowia Obecnych i Przyszłych pokolen; IUNiG PIG: Puławy, Poland, 2019.

48. Sapek, B. Zapobiegnie stratom i sekwestracji wegla organicznego w glebach łąkowych. Inżynieria Ekologiczna $2009,21,48-61$.

49. Antil, R.S.; Singh, M. Effects of organic manures and fertilization on organic matter and nutrients status of the soil. Arch. Agron. Soil Sci. 2007, 53, 519-528. [CrossRef]

50. Zimmer, J.; Roschke, M.; Schulze, D. Influence or different treatments of organic and mineral fertilization on yield, soil organic matter and N-balance of a diluvial sandy soil-Results after 45 years long-term field experiment P60 (Groß Kreuz, 1959-2003). Arch. Agron. Soil Sci. 2005, 51, 135-149. [CrossRef]

51. Ellmer, F.; Peschk, H.; Köhn, W.; Chmielewski, F.-M.; Baumecker, M. Tillage and fertilizing effects on sandy soils. Review and selected results of long-term experiments at Humboldt University Berlin. J. Plant Nutr. Soil Sci. 2000, 163, 267-272. [CrossRef]

52. Ellmer, F.; Baumecker, M. Soil Organic Matter of a Sandy Soil Influenced by Agronomy and Climate. In Proceedings of the International Meeting on Soil Fertility Land Management and Agroclimatology, Kusadasi, Turkey, 29 October-1 November 2008.

53. Józefaciuk, G. Zmiany właściwości powierzchniowych gleb i minerałów ilastych w procesach zakwaszania i alkalizacji. Badania modelowe. Instytut Agrofizyki PAN. Acta Agrophysica 1998, 15, 1-115.

54. Breymeyer, A.; Degórski, M.; Reed, D. Decomposition of pine-litter organic matter and chemical properties of upper soil layers: Transect studies. Environ. Pollut. 1997, 98, 361-367. [CrossRef] 